\title{
Geometry of emission in PSR 1929+10
}

\author{
Joanna M. Rankin \\ University of Vermont, Burlington, VT-05405, USA \\ N. Rathnasree \\ Raman Research Institute, Bangalore, 560080, INDIA
}

\section{Introduction}

Rankin (1990) attempted to distinguish between one- and two-pole interpulse models for PSR $1929+10$, and, despite the evidence to the contrary (Lyne \& Manchester 1988; Phillips 1990), argued that it was probably a two-pole interpulsar on the basis of its narrow components. If it is a two-pole interpulsar, its interpulse has just the right width to be a core component-that is, $2.45^{\circ} / P^{\frac{1}{2}}$, the angular width of the polar cap. However, PSR $1929+10$ was virtually the only well studied pulsar of any profile class which could not be comfortably fitted into the quantitative description of the conal emission geometry as discussed by Rankin (1993 a,b). The problem arose due to the narrow dimensions of the conal component pair in this pulsar, which do not fit well with the inner and outer cone properties followed by many pulsars. In order to explore this issue further, we use the technique of constructing partial "mode-separated" profiles corresponding to the primary and secondary polarisation modes.

\section{Polarisation mode separation}

Polarization-mode deconstruction is usually a complicated individual-pulse technique, but the highly polarised average profile of pulsar $1929+10$ suggests a simpler method. In the situation that the individual pulses are comprised only of one or the other fully polarised and orthogonal polarization modes,

$$
I_{\text {primary(secondary })}=\frac{I_{\text {total }} \pm L_{\text {total }}}{2},
$$

and we can reconstruct the modal profiles without requiring individual pulses.

The result of this procedure is depicted in Fig. 1(a) and (b) for both 430and $1414-\mathrm{MHz}$ observations. Here we have plotted only the total power because, under our assumption, both modes are fully polarised, and thus $I=L$ for each. The primary mode is plotted with a dashed curve, and the secondary with a dotted one (plotted $10 \times$ and $5 \times$ for clarity). There is much to see in these figures.

First, the great preponderance of the power falls in the primary mode at both 430 and $1400 \mathrm{MHz}$, representing $94.1 \%$ and $89.5 \%$ of the power at the two frequencies, respectively. Secondary-mode activity then divides into two 

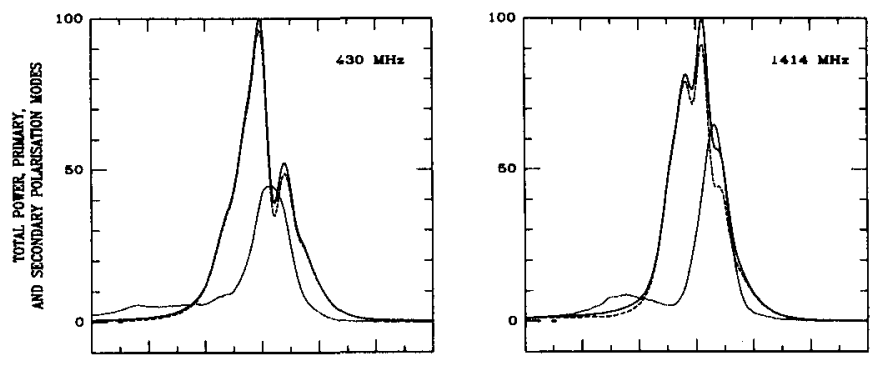

Figure 1. Empirical reconstruction of the partial polarisation-modal profiles at (a) $430 \mathrm{MHz}$, and (b) $1414 \mathrm{MHz}$.

regions, a weak one on the extreme leading edge of the profile and a stronger one which peaks near the intersection of components II and III. The primarymode profiles resemble the total profiles above, having three main (and perhaps five total) components. What is remarkable about the secondary-mode profiles is both their similar form and their utter lack of resemblance to the primarymode forms. There is simply no primary-mode feature on the leading edge of the profile where the secondary-mode profile first peaks, and its second "component" falls close to the minimum between comps. II and III and appears completely independent of them. These results are also confirmed by a more detailed sample by sample mode separation analysis based on the polarisation angle distribution (Rankin \& Rathnasree, in preparation).

This exceptionally clear decomposition of the polarisation modes in a highly polarised pulsar with a complex profile demands careful consideration. Yes, it does seem quite plausible in this case that all of the depolarisation results from the incoherent aggregation of power from the two orthogonal polarisation modes.

The 1929+10 MP profile provides us with a surfeit of features, a core component and what appear to be not two, but three conal component pairs. The two with the largest dimensions, the secondary-mode pair and the pair of features on the extreme edges of the average profiles(not shown), exhibit conal dimensions which are in agreement with the conal dimension scheme outlined by Rankin (1993 a,b). So, paradoxically, it is the narrowest pair comprised by the previously known conal components which appear to be anomalous.

Acknowledgments. This work supported in part by the US NSF (AST 89-17722). Arecibo Obs. is operated by Cornell Uni. under contract to NSF.

\section{References}

Lyne, A. G., \& Manchester, R. N. 1988, MNRAS, 234, 477

Narayan, R., \& Vivekanand, M. 1982, A\&A, 113, L3

Phillips, J. A. 1990, ApJ, 361, L57

Rankin, J.M. 1990, ApJ, 352, 247

Rankin, J.M. 1993a, ApJ, 405, 285

Rankin, J.M. 1993b, ApJS, 85, 145 